\title{
A maximum time approach to the computation of robust domains of attraction
}

\author{
Maurizio Falcone \\ Dipartimento di Matematica \\ Università di Roma "La Sapienza" \\ Piazzale A. Moro, 5 \\ 00185 Roma, Italy \\ falcone@caspur.it
}

\author{
Lars Grüne \\ Fachbereich Mathematik \\ J.W. Goethe-Universität \\ Postfach 111932 \\ 60054 Frankfurt a.M., Germany \\ gruene@math. uni-frankfurt.de
}

\author{
Fabian Wirth \\ Zentrum für Technomathematik \\ Universität Bremen \\ 28334 Bremen, Germany \\ fabian@math. uni-bremen.de
}

\begin{abstract}
We present an optimal control based algorithm for the computation of robust domains of attraction for perturbed systems. We give a sufficient condition for the continuity of the optimal value function and a characterization by Hamilton-Jacobi equations. A numerical scheme is presented and illustrated by an example.
\end{abstract}

\section{Introduction}

The domain of attraction of an asymptotically stable fixed point has been one of the central objects in the study of continuous dynamical systems. See e.g. $[6,15,3]$ for an analysis of the properties of the domains, [16] (cf. also the textbooks [9, 11]) for a characterization via PDE's and $[14,10,5,7]$ for computational approaches.

In the present paper we consider systems subject to deterministic time varying perturbations. We are interested in the set of points that is attracted to the fixed point regardless of the perturbation considered, under a local stability assumption which guarantees that it is reasonable to consider this set. This is what we call the robust domain of attraction. This subset of the domain of the unperturbed system $\dot{x}=f\left(x, a_{0}\right)$ is also studied in $[4,12,13]$. In particular an algorithm for the approximation of the robust domain of attraction based on ideas from optimal control is presented in [12]. There the robust domain of attraction is approximated by the sublevel sets of a sequence of optimal value functions.

In this paper we will present a different optimal control based approach using maximum time optimal control. Whereas the minimum time counterpart has been extensively investigated (see e.g. [1] and the references therein for the theory and [2] for the numerical treatment via Hamilton-Jacobi equations) this kind of optimal control problems is rarely found in the literature, presumingly also because of the lack of applications. In our context, however, these kind of problems appear naturally. In particular with this formulation we do not need an iterative construction of value functions but we just obtain the robust domain of attraction as the sublevel set of one particular optimal value function.

An important part of our approach is the characterization of the robust domain of attraction via a suitable first order partial differential equation. Another way to obtain such a 
characterization is by generalizing Zubov's classical method [16] to perturbed systems, see [4].

In Section 2 we will introduce the robust domain of attraction, in Section 3 we collect some basic facts about maximum time optimal control, in particular we introduce a condition for Lipschitz continuity of the optimal value function, and discuss the Kruzkov transform. Afterwards we give a numerical discretization scheme, and finally, in Section 5 present a numerical example.

\section{The robust domain of attraction}

We consider nonlinear differential equations subject to affine perturbations of the form

$$
\dot{x}(t)=f(x(t), a(t))=f_{0}(x(t))+\sum_{i=1}^{m} a_{i}(t) f_{i}(x(t))
$$

where $x \in \mathbb{R}^{n}, a \in \mathcal{A}=L^{\infty}([0,+\infty), A), A \subset \mathbb{R}^{m}$ is convex and compact, and the vector fields $f_{i}: \mathbb{R}^{n} \rightarrow \mathbb{R}^{n}$ are Lipschitz in $x$ for $i=0, \ldots, m$. Furthermore we assume uniform boundedness of $\|f(x, a)\|$ for all $x \in \mathbb{R}^{n}$ and all $a \in A$ and denote the bound by $M$. (Note that boundedness can always be achieved replacing $f(x, a)$ by $f(x, a) /\left(1+\left\|\sum_{i=0}^{m} f_{i}(x)\right\|\right)$ which changes the speed of the system but not the stability properties.) For an initial value $x_{0}$ at time $t_{0}=0$ and a fixed $a \in \mathcal{A}$ the solution will be denoted by $x\left(t, x_{0}, a\right)$.

We assume that the origin is a uniformly locally asymptotically stable equilibrium for the system (2.1) which can be characterized by means of class $\mathcal{K} \mathcal{L}$ functions: We call a function $\alpha:[0, \infty) \rightarrow[0, \infty)$ of class $\mathcal{K}$, if it satisfies $\alpha(0)=0$ and is continuous and strictly increasing, and we call a continuous function $\beta:[0, \infty)^{2} \rightarrow[0, \infty)$ of class $\mathcal{K} \mathcal{L}$, if it is of class $\mathcal{K}$ in the first argument and decreasing to zero in the second. Then the local stability assumption reads

$$
\begin{aligned}
& \text { there exists a class } \mathcal{K} \mathcal{L} \text { function } \beta \text { and a constant } r>0 \text { such that } \\
& \left\|x\left(t, x_{0}, a\right)\right\| \leq \beta\left(\left\|x_{0}\right\|, t\right) \text { for any } x_{0} \in B(0, r) \text { and any } a \in \mathcal{A} \text {. }
\end{aligned}
$$

In particular this implies $f_{i}(0)=0$ for $i=0, \ldots, m$. Now we can define our object of interest.

Definition 2.1 For the system (2.1) satisfying (H1) we define the robust domain of attraction as

$$
\mathcal{D}=\left\{x_{0} \in \mathbb{R}^{n}: x\left(t, x_{0}, a\right) \rightarrow 0 \text { as } t \rightarrow+\infty \text { for any } a \in \mathcal{A}\right\}
$$

In order to obtain a characterization of $\mathcal{D}$ being more suitable for our purpose consider a compact neighbourhood $\mathcal{N}$ of the origin with $\mathcal{N} \subset B(0, r)$. We introduce the "first hitting time" defined by $t(x, a):=\inf \{t>0: x(t, x, a) \in \mathcal{N}\}$. Note that by (H1) there exists $T>0$ independent of $x \in \mathbb{R}^{n}$ and $a \in \mathcal{A}$ such that $x(t, x, a) \in \mathcal{N}$ for any $t \geq t(x, a)+T$.

Lemma 2.2 The robust domain of attraction $\mathcal{D}$ satisfies

$$
\mathcal{D}=\left\{x \in \mathbb{R}^{n}: \sup _{a \in \mathcal{A}}\{t(x, a)\}<+\infty\right\} .
$$

Proof: Follows from [12, Lemma 4.1] by observing that the exponential stability assumed in this reference is not needed in the proof. 


\section{Maximum time optimal control}

The representation of $\mathcal{D}$ given in Lemma 2.2 leads to the following characterization: Consider a compact neighbourhood $\mathcal{N} \subset B(0, r)$ of the origin. Then we define the maximum time function by

$$
T(x):=\sup _{a \in \mathcal{A}} t(x, a) .
$$

It is immediate from Lemma 2.2 that $x \in \mathcal{D}$ if and only if $T(x)<\infty$. Furthermore it is easily seen that $T(x) \rightarrow \infty$ as $x \rightarrow \partial \mathcal{D}$, cp. e.g. [4].

Modifying the proof of [1, Chapter IV, Theorem 1.16] (there for the minimum time function) we obtain the following result on continuity of $T$.

Proposition 3.1 Assume $\partial \mathcal{N}$ is $C^{2}$, and

$$
\sup _{a \in A} f(x, a) \cdot \eta(x)<0
$$

for all $x \in \partial \mathcal{N}$, where $\eta(x)$ denotes the outward normal at $x \in \partial \mathcal{N}$. Then $T$ is Lipschitz continuous on $\mathcal{D}$.

Proof: Since $\partial \mathcal{N}$ and $A$ are compact and $f$ is continuous in $x$ and $a$ we find a constant $\xi>0$ such that

$$
f(x, a) \cdot \eta(x)<-2 \xi
$$

for all $x \in \partial \mathcal{N}, a \in A$. Define $d(x)=\operatorname{dist}(x, \partial \mathcal{N})$ for $x \notin \mathcal{N}$ and $d(x)=-\operatorname{dist}(x, \partial \mathcal{N})$ for $x \in \mathcal{N}$. Then the assumption on $\partial \mathcal{N}$ implies that $d$ is $C^{1}$ and thus $\eta(x)=\operatorname{Dd}(x)$ for all $x \in \partial \mathcal{N}$, implying that we can find an open neighbourhood $U$ of $\partial \mathcal{N}$ such that

$$
f(x, a) \cdot D d(x)<-\xi
$$

for all $x \in U, a \in A$. Furthermore, by boundedness of $f$ we find a neighbourhood $U_{1} \subset U$ of $\partial \mathcal{N}$ and a time $\tilde{t}>0$ such that $x\left(t, x_{0}, a\right) \in U$ for all $t \in[0, \tilde{t}]$ and all $x_{0} \in U_{1}$. Now consider an arbitrary $a \in \mathcal{A}$ and $x_{0} \in U_{1}$. Then, by Taylor expansion of $d$

$$
\begin{aligned}
d\left(x\left(t, x_{0}, a\right)\right) & =d\left(x_{0}\right)+\left(x\left(t, x_{0}, a\right)-x_{0}\right) \cdot D d\left(x_{0}\right)+o(M t) \\
& =d\left(x_{0}\right)+\int_{0}^{t} f\left(x\left(\tau, x_{0}, a\right), a(\tau)\right) \cdot D d\left(x_{0}\right) d \tau+o(M t) \\
& \leq d\left(x_{0}\right)-t \frac{\xi}{2}
\end{aligned}
$$

for all $t \in[0, \bar{t}]$ with $\bar{t} \in(0, \tilde{t}]$ sufficiently small. Thus denoting $t_{x_{0}}=\min \left\{\bar{t}, 2 d\left(x_{0}\right) / \xi\right\}$ we obtain $d\left(x\left(t_{x_{0}}, x_{0}, a\right)\right) \leq 0$ for all $x_{0} \in U_{1}$ with $d\left(x_{0}\right)<\bar{t} \xi / s$, hence $x\left(t_{x_{0}}, x_{0}, a\right) \in \mathcal{N}$ and consequently $T\left(x_{0}\right)<2 d\left(x_{0}\right) / \xi$.

Then the Lipschitz continuity of $T$ follows by the same argument as in [1, Chapter IV, Remark 1.7].

Remark 3.2 Finding a target meeting the assumptions of Proposition 3.1 is in general a nontrivial problem. If, however, the vector field $f_{0}$ is linearizable, the linearization is asymptotically stable, and the perturbation is sufficiently small (at least locally around the fixed point) we can always use a sublevel set of a quadratic Lyapunov function as our neighbourhood $\mathcal{N}$. 
Using analogous arguments as for the minimum time problem [1, Chapter IV.2] one can see that $T$ is the unique viscosity solution of the Hamilton-Jacobi equation

$$
\begin{cases}\inf _{a \in A}\{-f(x, a) \cdot D T(x)\}=1 & \text { on } \mathcal{D} \backslash \mathcal{N} \\ T(x)=0 & \text { on } \mathcal{N}\end{cases}
$$

Unfortunately, this function tends to infinity at the boundary of $\mathcal{D}$, hence $T$ is not suitable for computational purposes. Thus, for some real parameter $\delta>0$ we define the Kruzkov transform by

$$
v_{\delta}(x)= \begin{cases}1-e^{-\delta T(x)}, & x \in \mathcal{D} \\ 1, & x \notin \mathcal{D}\end{cases}
$$

It is immediate that $0 \leq v_{\delta} \leq 1$, and that it is the unique viscosity solution of the HamiltonJacobi equation

$$
\delta v_{\delta}(x)+\inf _{a \in A}\left\{-f(x, a) \cdot D v_{\delta}(x)\right\}=1 \text { on } \mathbb{R}^{n} \backslash \mathcal{N}
$$

with $\left.v_{\delta}\right|_{\mathcal{N}} \equiv 0$. Furthermore we have $\mathcal{D}:=\left\{x \in \mathbb{R}^{n} \mid v_{\delta}(x)<1\right\}$. Thus, the knowledge of $v_{\delta}$ gives us the desired set $\mathcal{D}$.

It is worth noting the effect of different $\delta>0$ on the function $v_{\delta}$. Since $T$ is bounded on any compact subset of $\mathcal{D}$, we obtain that $v_{\delta}(x) \rightarrow 0$ as $\delta \rightarrow 0$ uniformly on compact subsets of $\mathcal{D}$. This means the smaller $\delta>0$ is chosen, the steeper the function $v_{\delta}$ becomes at the boundary of $\mathcal{D}$. Thus computing $v_{\delta}$ with small parameters $\delta>0$ gives a sharp resolution of the boundary of $\mathcal{D}$ which can be used in the numerical approximation, cf. also Remark 4.1, below.

\section{The numerical scheme}

In this section we briefly sketch a numerical scheme for the maximum time problem. We use a straightforward modification of the scheme for the minimum time function from [2]. Note that alternatively one could use higher order approximations, see e.g. [1, Appendix A]. The convergence proof is obtained by straightforward modification of the arguments in those references.

Consider a compact set $\Omega \subset \mathbb{R}^{n}$ - our domain of computation — and a triangulation of $\Omega$ with $P$ simplices and $N$ vertices $x_{j}$. Fixing some time step $h>0$ we are looking for the function $\tilde{v}$ satisfying $\tilde{v}\left(x_{j}\right)=0$ for $x_{j} \in \mathcal{N}$ and

$$
\tilde{v}\left(x_{j}\right)=\sup _{a \in A}\left\{(1-\delta h) \tilde{v}\left(x_{j}+h f\left(x_{j}, a\right)\right)+h\right\},
$$

for all vertices $x_{j} \notin \mathcal{N}$ of the triangulation with linear interpolation on each simplex, setting $\tilde{v}(x)=1$ in the right hand side of (4.2) for $x \notin \Omega$. This implicit equation can be solved using iterative solvers, see e.g. [1, Appendix A] or [8]. As $h$ and the mesh size of the triangulation tend to 0 this function converges to $\left.v_{\delta}\right|_{\Omega}$, provided $\mathcal{D} \subset \Omega$. If this is not the case (e.g. when $\mathcal{D}$ is unbounded) we obtain an approximation of the relative robust domain of attraction

$$
\mathcal{D}_{\Omega}=\left\{\begin{array}{l|l}
x_{0} \in \Omega \mid \text { for each } a \in \mathcal{A}: \begin{array}{l}
x\left(t, x_{0}, a\right) \in \Omega \text { for all } t \geq 0 \text { and } \\
x\left(t, x_{0}, a\right) \rightarrow 0 \text { as } t \rightarrow \infty
\end{array}
\end{array}\right\} .
$$

Note that in general $\mathcal{D}_{\Omega} \neq \mathcal{D} \cap \Omega$ while clearly $\mathcal{D}_{\Omega} \subset \mathcal{D} \cap \Omega$. 
Remark 4.1 The adaptive grid scheme from [8] can be applied also to the calculation of solutions of (4.2). Since the local error estimates primarily refine the steep regions of the value functions this provides a fine resolution of $\partial \mathcal{D}$ for small $\delta>0$.

\section{A numerical example}

Our example is taken from [7, Example 6] with an additional perturbation term in the higher order nonlinearity.

$$
\begin{aligned}
& \dot{x}_{1}(t)=-x_{1}(t)+x_{2}(t) \\
& \dot{x}_{2}(t)=0.1 x_{1}(t)-2 x_{2}(t)-x_{1}(t)^{2}-(0.1+a(t)) x_{1}(t)^{3}
\end{aligned}
$$

Using the approach from the last section we investigated the robust domain of attraction for the origin. Here we used $\delta=1 / 1000, h=1 / 100$, and adaptive grids. Figure 5.1 shows the numerical approximations of the robust domains of attraction $\mathcal{D}$ for different sets of perturbation values $A$ by depicting the sublevel set $\tilde{v}(x)<0.8$. The set $\mathcal{N}$ has been chosen as an ellipse around the origin according to the Remark 3.2.
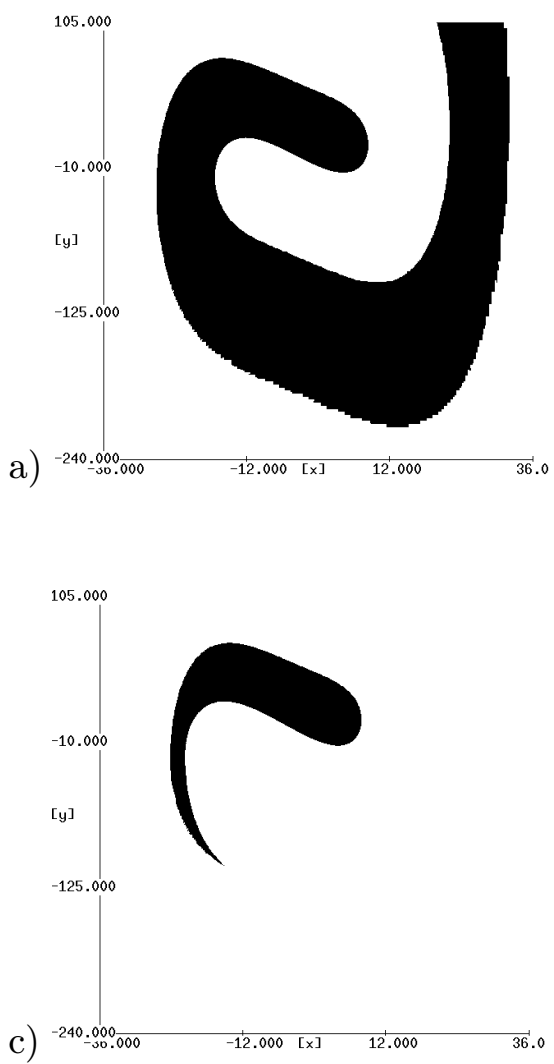
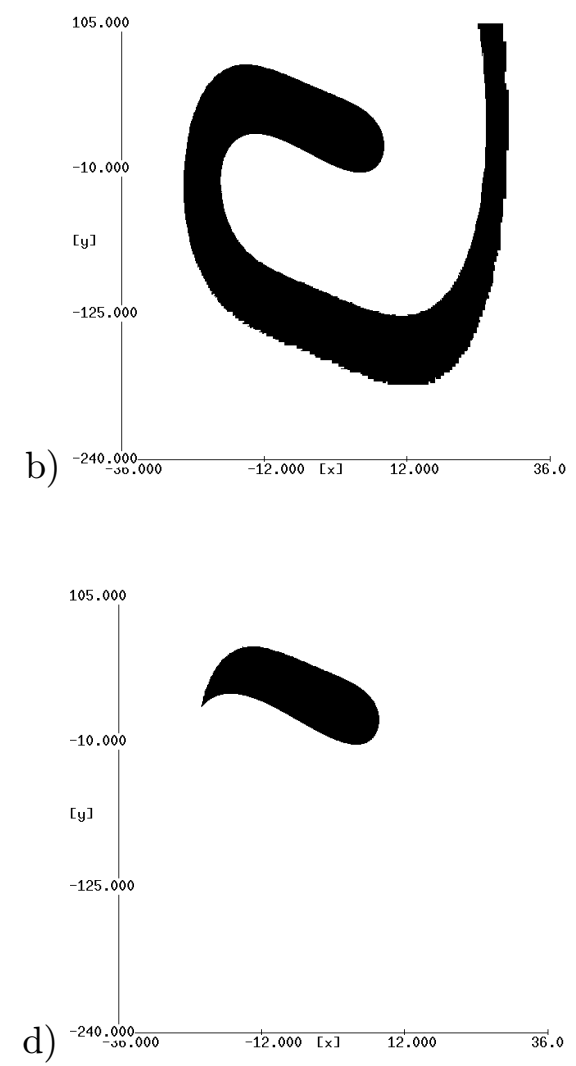

Figure 5.1: Approximation of $\mathcal{D}$ for a) $A=\{0\}$, b) $A=[-0.01,0.01]$, c) $A=[-0.02,0.02]$, and d) $A=[-0.03,0.03]$

Acknowledgements: Lars Grüne and Fabian Wirth would like to express their thanks for the hospitality of the Dipartimento di Matematica, Università di Roma "La Sapienza" where 
this research has been carried out. Research partially supported by DFG Grant GR1569/2-1, TMR Projects "Nonlinear Control" and "Viscosity Solutions and their applications", and DFG Priority Research Program "Ergodentheorie, Analysis und effiziente Simulation dynamischer Systeme".

\section{References}

[1] M. Bardi and I. Capuzzo Dolcetta, Optimal Control and Viscosity Solutions of HamiltonJacobi-Bellman equations, Birkhäuser, Boston, 1997.

[2] M. Bardi and M. Falcone, An approximation scheme for the minimal time function. SIAM J. Control Optim. 28 (1990), 950-965.

[3] N. Bhatia. On asymptotic stability in dynamical systems. Math. Syst. Theory 1 (1967), $113-127$.

[4] F. Camilli, L. Grüne, and F. Wirth. A Generalization of Zubov's method to perturbed systems. Preprint 24/99, DFG-Schwerpunkt "Ergodentheorie, Analysis und effiziente Simulation dynamischer Systeme"

[5] H.-D. Chiang, M. Hirsch, and F. Wu. Stability regions of nonlinear autonomous dynamical systems. IEEE Trans. Auto. Control 33 (1988), 16-27.

[6] C. Coleman. Local trajectory equivalence of differential systems. Proc. Amer. Math. Soc. 16(1965), 890-892. Addendum. Ibid. 17, 770 (1966).

[7] R. Genesio, M. Tartaglia, and A. Vicino, On the estimation of asymptotic stability regions: State of the art and new proposals. IEEE Trans. Auto. Control 30 (1985), 747-755.

[8] L. Grüne, An adaptive grid scheme for the discrete Hamilton-Jacobi-Bellman equation. Numer. Math. 75 (1997), 319-337.

[9] W. Hahn, Stability of Motion, Springer-Verlag, Berlin, 1967.

[10] N.E. Kirin, R.A. Nelepin and V.N. Bajdaev, Construction of the attraction region by Zubov's method. Differ. Equations 17 (1982), 871-880.

[11] H. K. Khalil. Nonlinear Systems. 2nd ed. Prentice-Hall, 1996.

[12] A. Paice and F. Wirth. Robustness analysis of domains of attraction of nonlinear systems, Proceedings of the Mathematical Theory of Networks and Systems MTNS98, pages 353356, Padova, Italy, 1998.

[13] A.D.B. Paice and F.R. Wirth. Robustness of nonlinear systems subject to time-varying perturbations, In F. Colonius et al. (eds.), Advances in Mathematical Systems Theory, Birkhäuser, Boston, 2000. to appear.

[14] A. Vannelli and M. Vidyasagar. Maximal Lyapunov functions and domains of attraction for autonomous nonlinear systems. Automatica, 21(1):69-80, 1985.

[15] F.W. Wilson. The structure of the level surfaces of a Lyapunov function. J. Differ. Equations 3 (1967): 323-329.

[16] V.I. Zubov, Methods of A.M. Lyapunov and their Application, P. Noordhoff, Groningen, 1964. 